\title{
La metodología GEEMPA, una trasformación en la forma de alfabetizar los niños del primer grado de primaria ${ }^{1}$
}

\author{
Hermelina Jaimes de Guerrero ${ }^{2}$ \\ Institución Nuestra Señora del Pilar, Pamplonita, Colombia \\ hermelina@gmail.com \\ Ruth Mayerly Guerrero Jaimes ${ }^{3}$ \\ Institución Nuestra Señora del Pilar, Pamplonita, Colombia \\ Ruth.guerrero@uniupamplona.edu.co
}

1 Artículo de investigación resultado del proyecto investigativo aplicado en el aula de clase.

2 Especialista en educación recreativa, Universidad de Pamplona. Docente Institución Nuestra Señora del Pilar, Pamplonita.

3 Magister en Ciencias Contables, Universidad de Los Andes, Mérida-Venezuela. Docente Universidad de Pamplona. 


\title{
La metodología GEEMPA, una trasformación en la forma de alfabetizar los niños del primer grado de primaria
}

\section{Resumen}

GEEMPA - Grupo de estudio sobre metodología de investigación y acción- es una nueva metodología flexible que busca alcanzar la alfabetización de un grupo de estudiantes de manera lúdica e incluyente. La autora de esta metodología es la doctora Esther Pillar Grossi, quien afirma que no existen problemas de aprendizaje, los maestros deben buscar nuevas estrategias de acuerdo a lo que piensan los niños y las niñas en los primeros años de su vida escolar. La presente investigación se encaminó a la implementación de esta metodología en los alumnos del primer grado de primaria del Colegio Nuestra Señora del Pilar del municipio de Pamplonita —Santander-, la cual consistió en la realización de un verdadero cambio en la forma de enseñar o de orientar los procesos de aprendizaje, se buscó alfabetizar a todos los niños sin importar el grado de dificultad que tuvieran. Se obtuvo que el juego en la clase es fundamental para el logro del aprendizaje. El juego de competición pone en acción una considerable suma de energías que difícilmente podrían ser movilizadas con una tarea escolar del tipo convencional. Actividades como la entrevista, fichas de aprendizaje, fichas didácticas, el juego como actividad, abecedarios y los cuadernos GEEMPA permitieron que se aplicara esta metodología, que produjo la entrega de resultados positivos en el proceso de aprendizaje de estos alumnos. Los niños fueron evaluados a través del modelo de entrevista y el análisis de la psicogénesis al final de cada periodo escolar; lo cual, en su fase final I, posibilitó obtener resultados positivos en sus procesos de aprendizaje.

Palabras clave: Investigación-acción, lúdica, alfabetización.

\section{GEEMPA methodology, a transformation in the way to alphabetize the children of first grade of primary}

\begin{abstract}
GEEMPA -Grupo de estudio sobre metodología de investigación y acción (by its Spanish definition) Study Group on Research and Action Methodology- is a new flexible methodology which goal is to alphabetize a group of students in an inclusive and ludic way. The author of this methodology, Dr. Esther Pillar Grossi, states that there are no learning issues, teachers are to find new teaching strategies in accordance to the way children see the world in their early years of school life. This research looks forward to the implementation of such a methodology on early grades of primary school at Nuestra Señora del Pilar school in Pamplomita municipality -Santander-. The investigation consisted in the development of a real change in the way of teaching or orienting the learning processes; children, no matter their learning issues, were alphabetized equally. playing in the classroom was identified as a key element for achieving learning. Contests triggers a big amount of energy that could hardly be produced by conventional homework. Interviews, flash cards, game, alphabets and GEEMPA notebooks allowed the implementation of this methodology, which produced positive results drawn from students' learning process. Children were approached by interviewing them and analyzing psichogenesis at the end of every academic term. The latter, on its final stage, allowed positive results from their learning process.
\end{abstract}

Keywords: Action-Reasearch, Ludics, alphabetization.

\section{A metodologia GEEMPA, uma trasformación na forma de alfabetizar os meninos do primeiro grau de primária (ensino fundamental}

\section{Resumo}

GEEMPA - Grupo de estudo sobre metodologia de investigação e ação- é uma nova metodologia flexível que procura atingir a alfabetização dum grupo de estudantes de maneira lúdica e incluyente. A autora desta metodologia é a doutora Esther Pillar Grossi, quem afirma que: não existem problemas de aprendizagem, os professores devem procurar novas estratégias de acordo com o que pensam os meninos e as meninas nos primeiros anos de sua vida escolar. A presente investigação encaminhou-se à implementação desta metodologia nos alunos do primeiro grau de primária do Colégio Nossa Senhora do Pilar do município de Pamplonita —Santander-, a qual consistiu na realização de uma verdadeira mudança na forma de ensinar ou de orientar os processos de aprendizagem, se procurou alfabetizar a todos os meninos sem importar o grau de dificuldade que tivessem. Obteve-se que o jogo na classe é fundamental para o lucro da aprendizagem. O jogo de competição põe em ação 
uma considerável soma de energias que dificilmente poderiam ser mobilizadas com uma tarefa escolar do tipo convencional. Atividades como a entrevista, fichas de aprendizagem, fichas didáticas, o jogo como atividade, cartilhas e os cadernos GEEMPA permitiram que se aplicasse esta metodologia, que produziu a entrega de resultados positivos no processo de aprendizagem destes alunos. Os meninos foram avaliados através do modelo de entrevista e a análise da psicogénesis ao final da cada período escolar; o qual, em sua fase final l, possibilitou obter resultados positivos em seus processos de aprendizagem.

Palavras chave: Investigação-ação, lúdica, alfabetização. 


\section{Introducción}

En 1970 [Esther Pillar] Grossi creó en Porto Alegre, junto con medio centenar de profesores, el Grupo de estudios sobre educación, metodología de investigación y acción —-GeEMPA, por sus siglas en portugués—, que ideó un método del mismo nombre que revolucionó la educación pública en Brasil desde 1982 y que se emplea, con muy buenos resultados, a partir del 2005 en Colombia. El método ha beneficiado a miles de estudiantes de clases populares o con limitaciones cognitivas como el síndrome de Down (Portafolio, 28 de septiembre de 2012).

Como menciona la doctora Grossi ${ }^{4}$ (2012): «La gran innovación que estamos introduciendo en escuelas públicas de Brasil es una manera de enseñar basada en nuevas contribuciones científicas sobre el aprender» (p. 13).

La autora explica que el descubrimiento de Piaget:

[...] que afirma que entre la percepción y el conocimiento hay una construcción, cambió radicalmente la enseñanza porque nos aclaró que aprender es un proceso interno de cada persona y no algo que se ofrece listo desde afuera, como se cree hasta hoy la enseñanza convencional (Grossi, 2012, p. 11).

Esta metodología emplea principalmente la lúdica en la enseñanza para activar procesos de producción escrita, lectura comprensiva, pensamiento lógicomatemático y convivencia pacífica, superando a la metodología tradicional que impulsa la lectura y escritura automática o memorística; asimismo, enfatiza en el cómo y en el por qué, lo que garantiza un proceso de alfabetización exitoso. El GEEMPA no enseña la respuesta, sino la pregunta: «Eso cambia radicalmente la enseñanza, suministra nuevas posibilidades de éxito y se convierte casi en la única posibilidad para los alumnos de las escuelas públicas»(Grossi, 2005, p. 12). Los resultados significativos de su aplicación son las elevadas tasas de aprendizaje de los alumnos. Según Grossi (2005), «se logra alfabetizar el 100 por ciento de los alumnos entre tres y cinco meses» (p. 11).

En el año 2012, el Ministerio de Educación y la Corporación Conprender deciden realizar una prueba piloto de capacitación en esta metodología de los docentes del grado de primero de primaria, a nivel nacional, para alfabetizar masivamente, dados los alcances demostrados. Es así como la institución educativa Nuestra Señora del Pilar del municipio de Pamplonita concluye el deber de su aplicación

4 "En Colombia, Grossi trabaja con la Corporación Conprender (con n) y con el Ministerio de Educación. Instruyó a profesores de 10 departamentos en el 2005 y su método ha beneficiado a más de 15.000 estudiantes, sobre todo, de colegios oficiales, de acuerdo con la educadora Sonia Matillana, directora de Conprender" (Portafolio, 28 de septiembre de 2012). 
bajo el propósito de mejorar los procesos de lectoescritura, producción de textos y comprensión lectora, lo que arrojó resultados satisfactorios a partir de la aplicación de esta metodología. Debido al seguimiento realizado a estos niños y niñas se pudo demostrar el gran avance en comprensión lectora y de escritura que desarrollaron, comparado con lo logrado con la metodología tradicional, según lo expuesto por la docente encargada del año primero de primaria en los últimos seis años en esta institución.

\section{Por qué aplicar la metodología GEEMPA a los jóvenes del primer grado de primaria}

Trabajar una nueva metodología exige un verdadero cambio en las formas de enseñar o de orientar los procesos de aprendizaje. Cambiar paradigmas no es nada fácil, pero esto es posible dependiendo de la actitud y el deseo por hacer las cosas cada vez mejor; especialmente, se trata de vivir la profesión de maestros a plenitud. La misión es clara cuando se decide tomar el camino de la docencia y por eso no es difícil emprender el nuevo reto de aplicación de una metodología que exige un poco más de esfuerzo, en la que los resultados Ilenarán de satisfacción y se alcanzarán más rápido de lo esperado respecto a cualquier trabajo tradicional realizado en las aulas.

Como menciona Ruiz (2014): «Esta didáctica pretende garantizar el acceso al mundo letrado de niños, niñas, jóvenes y adultos que habían sido excluidos del sistema educativo por sus condiciones cognitivas» (p. 6.). Se trata de cambiar el modelo pedagógico tradicional de los alumnos del primer grado de básica primaria -método que en muchas ocasiones pierde sentido dentro de las instituciones educativas si no se cuenta con estas destrezas desde preescolar - para transitar a otro modelo pedagógico que facilite el proceso de aprendizaje de manera lúdica y creativa, que permita un trabajo paralelo de lectura, escritura, matemáticas; objetivos perseguidos por esta nueva didáctica.

De acuerdo a Giraldo (2016): «Gracias a esta didáctica, las docentes, mediante el juego y un material especial para implementar sus clases, han logrado que sus estudiantes avancen significativamente en sus procesos de aprendizaje, pudiendo notar sus resultados en poco tiempo» (p. 1).

Con el modelo tradicional se venía detectando que a muchos alumnos se les dificultaba leer y escribir bien, y tenían problemas de comprensión lógica, lectora y de memorización. Al interactuar con ellos y ellas para tratar de compartirles un cuento, se notó de inmediato que su participación al respecto no podía llevarse a cabo dadas las manifiestas deficiencias que tenían por su desconocimiento del abecedario, la nula construcción de palabras o frases sencillas y la incapacidad de 
formar sílabas con consonantes y vocales. Pero, además, algunas(os) manifestaron por momentos un rechazo al uso del cuaderno como único espacio sobre el cual podían practicar sus ejercicios.

GEEMPA — Grupo de estudios en educación, metodología de investigación y acciónpretende quebrar muchos de los esquemas de la escuela tradicional y configurarse como un método sobre el aprendizaje, donde se refiere al deseo como un gran motor del aprendizaje. Una de las prioridades de esta metodología es generar interés y motivación en los niños por la lectura y escritura para evitar el fracaso y repitencia escolar (Cervera y Grimaldo, 2008).

Con este nuevo modelo pedagógico se pretende que los alumnos del primer grado lean y escriban en menos tiempo y tengan gran capacidad lectora y lógica, que desarrollen habilidades y destrezas en las diferentes áreas del conocimiento, de acuerdo a su etapa de desarrollo, a través de actividades lúdicas que les permiten ir jugando y aprendiendo.

\section{El contexto}

El Colegio Nuestra Señora del Pilar es una institución educativa oficial ubicada en el casco urbano del municipio de Pamplonita, Norte de Santander. Cuenta con aproximadamente cuatrocientos cincuenta estudiantes, la mayoría de estrato 1 y 2 . El grado primero, en los últimos tres años, ha estado conformado por un número de treinta estudiantes, la mayoría procedentes de las veredas de alrededor del casco urbano, muchos de ellos de escasos recursos, pero con gran interés por aprender, por eso hacen un gran esfuerzo por salir adelante y estudiar.

\section{En qué consiste la metodología GEEMPA}

GEEMPA nació hace treinta y ocho años en Brasil y toma su nombre de la onG que lo implementó, inicialmente, para la enseñanza de las matemáticas en población de bajos recursos de las favelas marginadas. GEEMPA es una sigla que quiere decir Grupo de Estudio sobre Educacion. Esther Grossi, su creadora, toma técnicas del «postconstructivismo», el cual categoriza actividades de cómo un niño aprende a leer y escribir mediante materiales didácticos. Tal metodología consiste en valorar inicialmente el nivel de lectura y escritura de cada estudiante en tres niveles: presilábico, silábico y alfabético, y se desarrolla por medio de un material didáctico que incluye juegos, textos de cuentos, cartas y fichas de trabajo; a su vez, se basa en las propias experiencias del estudiante y su interacción con los demás, lo que permite ir evaluando el grado de aprendizaje que va logrando a través de su año escolar.

Para Lev Vigotsky, filósofo y sicólogo ruso, uno de los teóricos más destacados de la sicología del desarrollo: «El lenguaje es la herramienta cultural de aprendizaje por 
excelencia. El individuo construye su conocimiento porque es capaz de leer, escribir, preguntar a otros y a sí mismo sobre aquellos asuntos que le interesan» (como se cita en Conprende, 2010 p. 39). Vigotsky planteó que existe en cada uno una Zona de Desarrollo Próximo, esto es, un espacio en el que, gracias a la interacción y la ayuda de otros, una persona puede trabajar y resolver un problema o realizar una tarea de una manera y con un nivel que no sería capaz de tener individualmente.

Las investigaciones realizadas por Piaget, Wallon, Ferreiro, Taberosky, Vergnaud, Pain y muchos otros han servido de base para formular un modelo que orienta a los docentes para lograr la alfabetización y superar el mito de los mal Ilamados problemas de aprendizaje (Conprende, 2010, p. 39).

La teoría piagetiana explica esencialmente el desarrollo cognoscitivo del niño haciendo énfasis en la formación de estructuras mentales.

Igualmente, es importante el concepto de intersubjetividad, que está implícito en el planteamiento de Wallon, en el estudio de la relación con el otro. Vale la pena mencionar a Emilia Ferreiro y Ana Taberosky, por su aporte sobre La Psicogénesis de la lengua escrita: Comprensión del proceso evolutivo de la adquisición de la lengua escrita; a Gerard Vergnaud, por la teoría sobre los campos conceptuales, y a Sara Pain, por sus investigaciones sobre la construcción del conocimiento desde lo que ella ha denominado la función de la ignorancia, el cual es:

[...] el eje conceptual del modelo postconstructivista diseñado por la doctora Esther Pillar Grossi en Brasil, que no solamente nos presenta la teoría sino que va más allá y diseña el cómo, o sea, la didáctica que permite garantizar el acceso al mundo letrado de niños, niñas, jóvenes y adultos que habían sido excluidos de ese saber (Conprende, 2010 p. 121).

Esta propuesta didáctica está sustentada en varias premisas o postulados, el más importante de ellos es que TODOS PUEDEN APRENDER. Esto contradice la práctica pedagógica actual, que está construida sobre falsos presupuestos empiristas y que no tienen en cuenta la lógica del desarrollo cognitivo del estudiante. La primera línea que caracteriza al GEEMPA es la determinación de las instancias que interfieren en el aprendizaje; tales son: la instancia lógica, que se ocupa de la adquisición de los conocimientos de la realidad objetiva; la instancia simbólica, que se ocupa de la organización de la realidad subjetiva de cada persona, de aquello que caracteriza el mundo interior personal, el de los deseos; la instancia del cuerpo, lugar donde se realizan las percepciones, los movimientos, los afectos que se interiorizan para el funcionamiento de las dos instancias anteriores; y la instancia del organismo, que es la materia prima para la construcción del cuerpo. Las cuatro instancias son responsables de la adquisición del conocimiento. 


\section{Metodología}

GEEMPA es una metodología que implementa actividades precisas y reiterativas que, en el proceso de enseñanza-aprendizaje de la lectoescritura, trasciende lo memorístico, haciendo el análisis de cada palabra en torno a las letras y la cantidad que la conforman, las que la inician y terminan, su secuencia y demás posibilidades. Por medio de GEEMPA se tiene en cuenta la lógica del aprendizaje de los estudiantes y no la de los contenidos a enseñar, es decir, la idea es tener en cuenta la experiencia y formas de interacción que con el conocimiento muestran los niños y las niñas del primer grado a partir de lo que el material dispuesto les vaya generando en el mejoramiento de su proceso de lectura y escritura. Se trabajó teniendo en cuenta el contexto semántico de letras, palabras y textos, en letra imprenta, dispuestos en franjas de cartulina para que los niños las identifiquen, caractericen y asocien.

Como la idea de la metodología GEEMPA es que los niños y las niñas en grupo construyan su propio conocimiento por medio de las experiencias y desarrollen actitudes y capacidades que les permitan desenvolverse activamente en interacción con ellos mismos y con la realidad — por medio de la apropiación e interiorización de sus aprendizajes vivenciales-, cada actividad que aquí se propuso se dio en forma colectiva, escuchando en forma ordenada y respetuosa, aportando y ayudando cada uno y cada una en el análisis de lo que poco a poco y paso a paso se va generando. De otra parte, cuando cada niño o niña expone lo que la etiqueta o el juego le posibilita descubrir y construir, los demás están atentos para considerar si va por buen camino lo que está realizando; en caso de que no, se le ayuda a ver qué le pasó y por dónde debe direccionar el ejercicio a fin de que lo saque adelante y verifique esta manera de apropiarse del conocimiento; en caso de que sí, se le muestra como ejemplo a seguir por la consecución y desarrollo de su nueva habilidad.

\section{Propósitos a desarrollar de la didáctica GEEMPA en los niños de primer grado}

De acuerdo a la metodología GEEMPA, se busca superar la enseñanza tradicional de la lectura y escritura y cumplir los siguientes objetivos:

- Todos los niños y niñas aprenden según sus esquemas mentales y sus niveles de psicogénesis de lectura y escritura.

- Evitar la deserción escolar.

- Hacer del juego la principal herramienta pedagógica.

- Garantizar la alfabetización: lectura comprensiva y producción escrita.

- Formar niños y niñas competentes para la vida. 


\section{Desarrollo de la metodología GEEMPA}

Para el desarrollo de la metodología es necesario desarrollar las siguientes estrategias:

\section{Conformación de grupos de docentes}

Los grupos de estudio son un apoyo invaluable dentro de la didáctica.

Cada maestro tiene una percepción específica de un tema o asunto de acuerdo a sus propias experiencias y su bagaje cultural y emotivo. Por tanto, contar con un grupo de estudio ayuda a aterrizar los temas, la didáctica y su abordaje.

\section{La clase-entrevista}

Es un instrumento útil para caracterizar la red de hipótesis durante el proceso de afabetización y les permite a los niños expresarse libremente sobre un acontecimiento elegido a su gusto, del cual el maestro va tomando los elementos para planificar las actividades coherentes con los esquemas de pensamiento de los estudiantes. Esta entrevista se realiza al inicio de año y después de cada periodo con el propósito de conocer el avance en su proceso de aprendizaje.

\section{Organización de grupos según su psicogénesis}

La psicogénesis consiste en entender qué tanto los niños pueden asimilar en su proceso de aprendizaje. Esta actividad se debe realizar cuando se requiera como actividad de profundización o refuerzo. Se establece, por ejemplo, un día en la semana, para así detectar y planear actividades exclusivas con base en las necesidades específicas de los estudiantes, ubicados según su avance en la escalera psicogenética de lectura y escritura.

\section{Actividades de rutina}

- Canción de bienvenida.

- Entrega de las escarapelas.

- Asistencia y autoasistencia.

- Canción del alfabeto.

- Lectura - contrato didáctico-.

- Lectura - contexto semántico-.

- Juego de familiarización.

- Juego de aprendizaje.

- Ficha didáctica.

- Actividad de escritura. 


\section{Cuadernos GEEMPA}

Cuaderno de producción textual

Este cuaderno tiene como objetivo el seguimiento del avance en términos de escritura y lectura del o la estudiante. Es muy importante que este cuaderno permanezca en el aula, el estudiante no debe llevárselo para la casa, pues lo padres tienden a corregirlo, criticarles, borrarles o completarles lo que no terminaron.

El cuaderno es de uso exclusivo del estudiante. El maestro le pide que lea lo que escribió ahí y va siguiendo la lectura; inicialmente hace la petición de manera informal y de memoria para que recuerde lo que escribió e invente. A medida que va avanzando en la didáctica, hay mayor coherencia y concordancia.

En el cuaderno, EL DOCENTE no debe corregir, marcar, tachar, borrar, ni contaminar de ninguna manera su trabajo de producción; con el tiempo, el propio estudiante al leer encontrará sus errores iniciales y decidirá bajo su criterio si los corrige o los deja así.

\section{Cuaderno-diccionario}

Este es un cuaderno que está dispuesto, en sus páginas marcadas con el alfabeto, como si fuera un diccionario. Cada Rótulo va en letra imprenta, cursiva, MAYúscula y minúscula. En el cuaderno los estudiantes escriben las nuevas palabras que conocieron, palabras significativas para él y las palabras alfabetizadoras de cada uno de los Contextos Semánticos Trabajados. Éste se lleva con mucha pulcritud, muy bien escrito, con ortografía y letra legible. En este cuaderno el maestro puede pedir a los estudiantes que hagan correcciones cuando está mal escrita una palabra.

\section{Cuaderno de tareas}

En este cuaderno se consignan palabras, frases, y se puede hacer seguimiento a las fichas didácticas trabajadas en clase.

\section{Abecedarios}

Los abecedarios, son ayudas didácticas para reafirmar las actividades planeadas.

Abecedario de pared

Este es un abecedario que se hace del tamaño de una hoja de papel bond o kraft. Allí se ubica de manera vertical todo el alfabeto, tanto en letra mayúscula como minúscula, y se hacen cuadros dejando horizontalmente espacios en blanco para que en las distintas actividades los estudiantes ubiquen las palabras según su letra inicial y las escriban ahí. 


\section{Abecedario de hoja}

Es una hoja donde se encuentra en forma vertical todo el alfabeto. En las actividades planeadas dispuestas por la docente, se pueden utilizar para ubicar las palabras alfabetizadoras de acuerdo a su letra inicial. Tales hojas también se usan para realizar dictados, o que los estudiantes busquen palabras y las ubiquen ahí según el criterio e imaginación del docente.

\section{Fichas didácticas}

El trabajo de un contexto semántico implica actividades de lectura y escritura trabajadas en las fichas didácticas, también denominadas fichas de actividades o guías de trabajo. Éstas son elaboradas por los docentes para ser desarrolladas por los estudiantes. Generalmente, las fichas didácticas son fotocopias que después de su desarrollo el estudiante guarda en una carpeta personal.

Las fichas didácticas son un recurso imprescindible dentro de la didáctica y su función principal es reforzar o reafirmar el aprendizaje que se obtiene del juego; se realizan según las necesidades del grupo y la meta diaria.

\section{Resultados}

Después de aplicada esta metodología y realizadas las clases-entrevistas en los cuatro periodos escolares, se analizaron los resultados arrojados por las entrevistas aplicadas a los niños y niñas del primer año de primaria de la institución educativa Nuestra Señora del Pilar.

Al inicio del año se elaboró la psicogénesis de lectura y escritura de acuerdo a los resultados que arrojaron las entrevistas.

Figura 1. Modelo de psicogénesis para medir el proceso de aprendizaje de lectoescritura. Clase-entrevista de inicio de año

PRIMERA ENTREVISTA - ESTRUCTURA DE CUATRO PALABRAS Y UNA FRASE

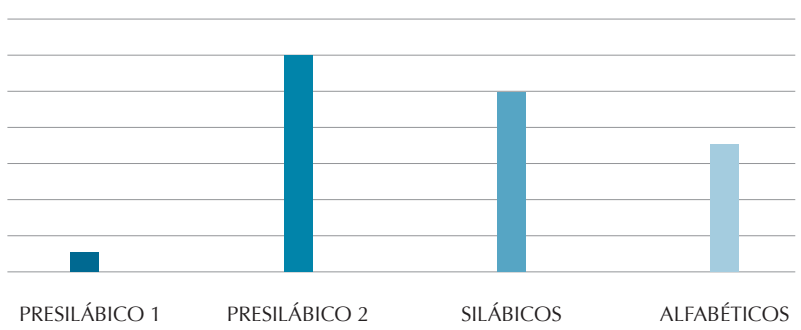


Tema Libre

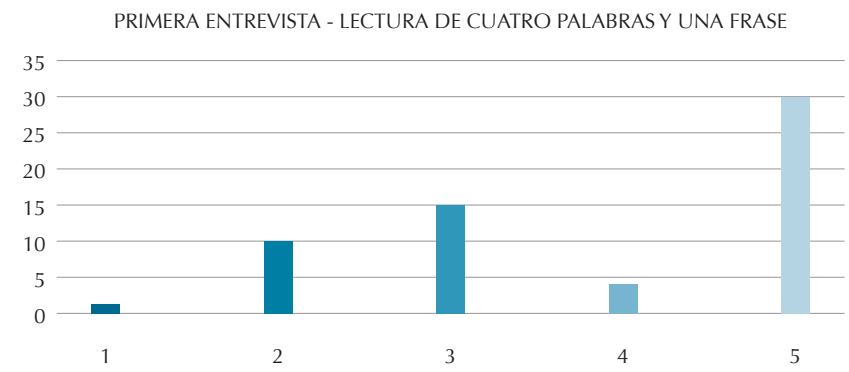

ESCALERA DE LA PSICOGÉNESIS

III TAREA

Escritura de las cuatro palabras y una frase.

Fecha: 21-23 de Enero

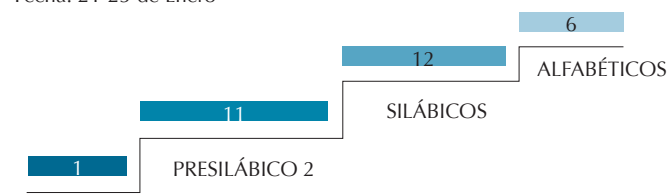

PRESILÁBICO 1

VTAREA

Lectura de las cuatro palabras y una frase. Fecha: 21-23 de Enero

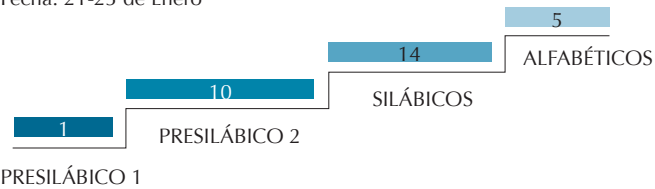

Fuente: elaboración propia (2016).

En el segundo periodo se realizó la entrevista para evaluar el grado de avance en el aprendizaje a esa fecha.

Figura 2. Modelo de psicogénesis para medir el proceso de aprendizaje de la lectoescritura. Clase-entrevista, segundo periodo

SEGUNDA ENTREVISTA - LECTURA DE CUATRO PALABRAS Y UNA FRASE

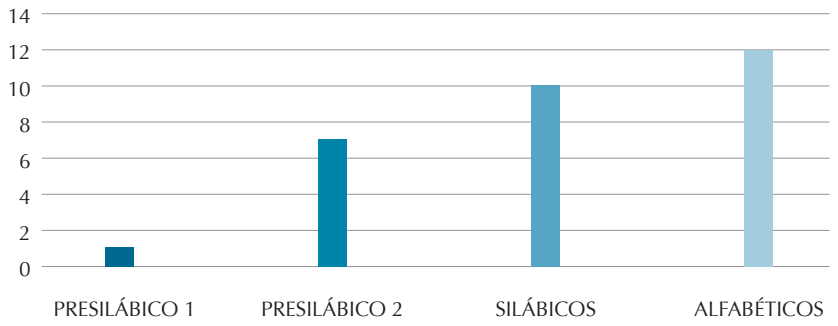


La metodología GEEMPA, una trasformación en la forma de alfabetizar los niños del primer grado de primaria | Hermelina Jaimes de Guerrero | Ruth Mayerly Guerrero Jaimes |

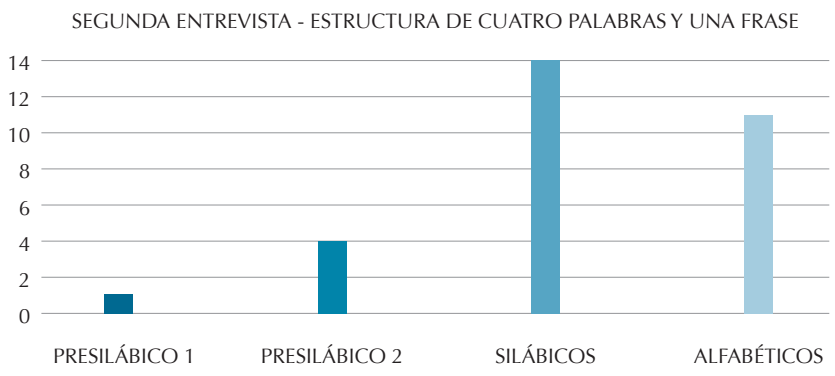

Fuente: elaboración propia (2016).

Una vez finalizado el tercer periodo, se procedió a realizar nuevamente la entrevista para continuar evaluando el avance obtenido por los niños y niñas del grado primero.

Figura 3. Modelo de psicogénesis para medir el proceso de aprendizaje de la lectoescritura.

Clase-entrevista, tercer periodo

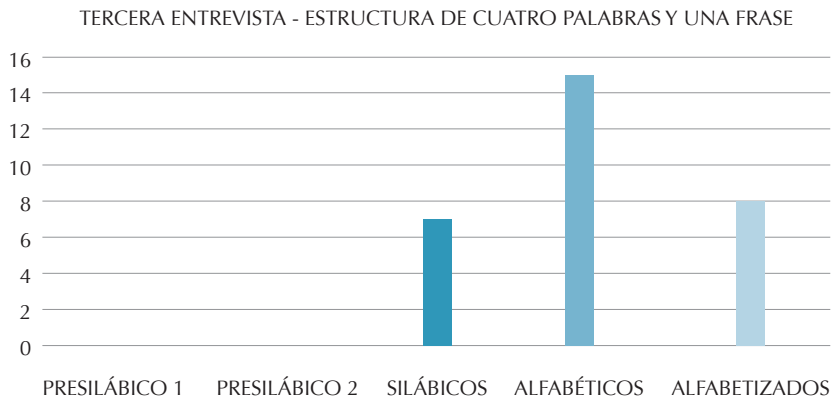

Fuente: elaboración propia (2016).

Al final del año, se realizó una cuarta entrevista que correspondió al último periodo, la cual permitió obtener resultados definitivos sobre la aplicación de la metodología y así poder evaluar el grado de desarrollo de los niños y niñas, del grado primero de primaria del Colegio Nuestra Señora del Pilar, para aprender a leer y escribir. 
Tema Libre

Figura 4. Modelo de psicogénesis para medir el proceso de aprendizaje de la lectoescritura. Clase-entrevista, Final de año

CUARTA ENTREVISTA - LECTURA DE CUATRO PALABRAS Y UNA FRASE

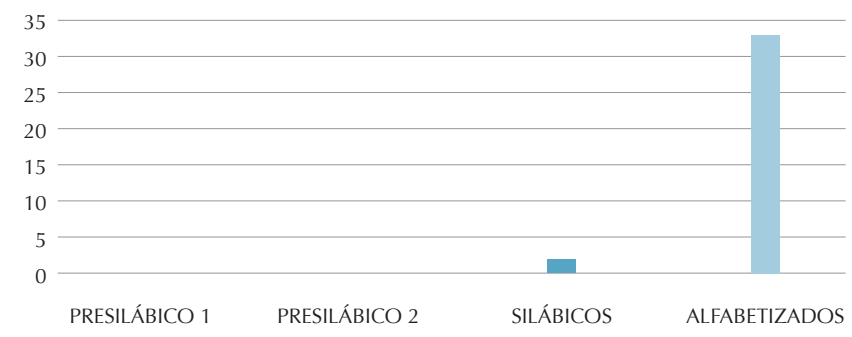

CUARTA ENTREVISTA - ESCRITURA DE CUATRO PALABRAS Y UNA FRASE

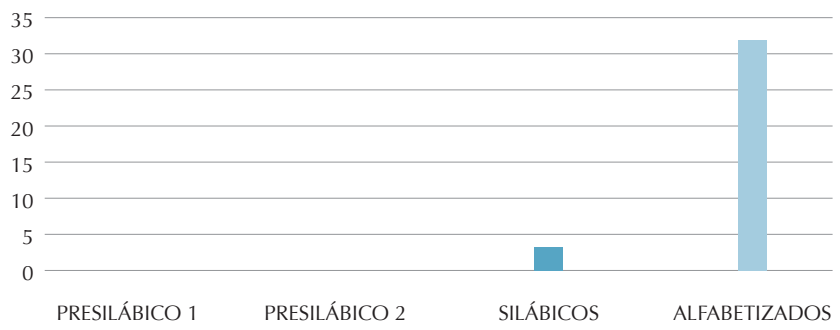

ESCALERA DE LA PSICOGÉNESIS

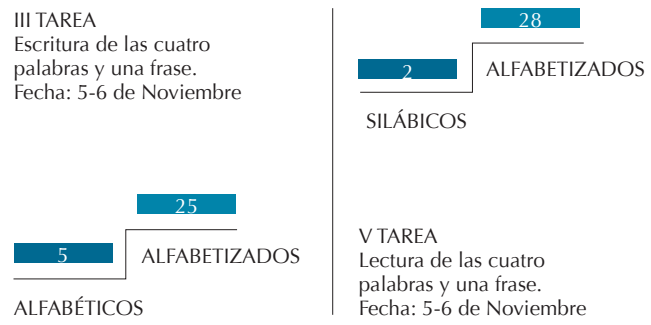

Fuente: elaboración propia (2016).

Esta nueva didáctica permitió realizar un trabajo paralelo de lectura y escritura hasta llegar con éxito a la alfabetización, ya que el 93\% de los niños y niñas del primer grado lograron el objetivo planteado.

Asimismo, esta metodología facilitó que los niños y niñas desarrollaran habilidades para: 
- Reconocer en la lectura de algunos textos ideas que los invitan al respeto de sus semejantes.

- Identificar el mejoramiento de habilidades comunicativas, lo que incrementa sus posibilidades de hacer nuevas amistades.

- Leer y escribir el nombre de sus compañeros y compañeras.

\section{Conclusiones}

La implementación de la metodología GEEMPA, empleada en los niños y niñas del primer grado de primaria del colegio Nuestra Señora del Pilar del municipio de Pamplonita, permitió comprobar la bondad y eficacia de este método a través del cual, en la actualidad, los niños que se encuentran cursando el grado cuarto de primaria han demostrado tener una mayor capacidad de desarrollo en la producción textual y comprensión lectora, resultados que se vieron reflejados en los diferentes concursos en los que los niños - con los que se comenzó la aplicación de esta metodología en el año 2013- obtuvieron la más alta calificación.

Al ejecutar las diferentes actividades planteadas por esta metodología, los niños juegan y al mismo tiempo aprenden; esto les posibilitó estar más interesados y desinhibidos, facilitando un mejor desarrollo su proceso lectoescritor.

Esta nueva metodología exige un verdadero cambio en las formas de enseñar y de orientar los procesos de aprendizaje. A través de la autoconstrucción de conocimiento, sobre todo mediante el elemento lúdico, se ayuda a entender con más satisfacción cada actividad planeada. Igualmente, la metodología GEEMPA exige al educador pensar, crear estrategias, actividades y espacios que le permitan adentrar a los estudiantes al mundo de la lectura y la escritura, lo que facilita que ellos construyan su propio conocimiento. 


\section{Referencias}

Cervera, S., y Milena, C. (2008). Geempa como metodología dinamizadora en el proceso de aprendizaje de la lectura y la escritura en el grado primero de primaria [Tesis de pregrado]. Bogotá: Corporación Universitaria Minuto de Dios.

Conprende. (2010). Apoyo didáctico para alfabetizadores. Bogotá: Corporación de capacitación y apoyo para la discapacidad —Conprende-.

Giraldo, G. (2016). La Didáctica Geempa, Una Alternativa Para EnseñarY Aprender De Manera Diferente. Recuperado de http://www.vocerodelcafe.com/metro/la-didactica-geempauna-alternativa-para-ensenar-y-aprender-de-manera-diferente

Portafolio. (28 de septiembre de 2012). Método GEEMPA, una forma diferente de aprender. Recuperado de http://www.portafolio.co/tendencias/metodo-geempa-forma-diferenteaprender-97184

Ruíz, J. (2014). Implementación de la Metodología GeEmPA en la lectoescritura musical. Revista Paideia Surcolombiana, 19, 35-43.

Recibido: 19 de agosto 2016

Aceptado: 27 de octubre 2016

Cómo citar: Jaimes, H., y Guerrero, R. (2017). La metodología GEemPA, una trasformación en la forma de alfabetizar los niños del primer grado de primaria. Praxis Pedagógica, 20, 85-100 